\title{
Loss of Renal Function After Retrograde Ureteral Placement of an Allium Stent for Severe Ureteral Stricture
}

\author{
Stefano Salciccia, MD, Alessandro Sciarra, MD, Milena Polese, PhD, Alpi Giorgio, MD, \\ Martina Maggi, MD, Alessandro Gentilucci, MD,' Mauro Ciccariello, MD, Giuseppe D'Eramo, \\ Houssain Shahabadi, MD, Silvia Lai, MD, and Gian Piero Ricciuti, MD ${ }^{1}$
}

\begin{abstract}
Background: Ureteral strictures are a recurrent chronic condition that leads to severe side effects and poor quality of life. Management of ureteral stricture is a great challenge for urologists and no specific guidelines exist. Retrograde Allium ${ }^{\circledR}$ ureteral stent (AUS) is a newly developed ureteral stent to treat either bulbar urethral or ureteral stenosis.

Case Presentation: We describe a case of a 74-year-old Caucasian adult male presenting with a severe ureteral stricture secondary to an ureteroscopy for stone disease. Treatment with retrograde AUS placement produced a complete loss of renal function after 36 months, probably because of the development of a long achalasic stretch of the ureter.

Conclusions: AUS is a new and promising device for the treatment of ureteral stenosis. However, a lack of standardization of the technique recommends a close instrumental follow-up after the procedure to decide the optimal time for stent removal.
\end{abstract}

Keywords: ureteral stent, ureteral stricture, stone disease complications

\section{Introduction and Background}

$\mathbf{U}$ RETERAL STRICTURE IS a well-known complication after endoscopic procedures in the upper urinary tract. ${ }^{1}$ However, the exact mechanism for which stenosis develops is not well known. Some studies have revealed that in case of impacted calculi, ureteral perforation during the procedure is related to a high rate of stricture development $(90 \%$ to $95 \%){ }^{2}$ Damage to the ureteral mucosa can also trigger stricture formation. Fibrinous exudates produced by the mucosa precipitate into the injured area, stimulating adherence and, finally, stricture formation. ${ }^{3}$ Management of ureteral strictures is a great challenge for urologists.

To date, we have no specific recommendations on how to treat ureteral stenosis. Traditionally, the open surgical treatment of ureteral strictures included ureteroureteral anastomosis, ileal substitution, autotransplant, or nephrectomy. With the technological evolution, endoscopic treatments such as balloon dilatation, cold knife incision, and laser endoureterotomy are more commonly used, including the insertion of new types of ureteral stent. ${ }^{4}$ The Allium ${ }^{\circledR}$ ureteral stent (AUS) is a newly developed ureteral stent, which can be inserted in structures of small diameter and spontaneously expands and preserves a large caliber. The stent is coated with a biochemical copolymer that prevents tissue ingrowth and incrustation. ${ }^{5}$ The stent can be inserted endoscopically with an intraoperative $\mathrm{X}$-ray guidance after dilatation of the stricture and then it can be removed in a time range from 6 to 12 months. We present the case of a man with a complete loss of left kidney function after positioning of the AUS for an indwelling time of 36 months.

\section{Case Presentation}

On March 2013, a 74-year-old Caucasian man with a history of stone disease was evaluated in our urologic department for left side pain. Medical history included only hypertension under control with beta-blockers. Ultrasonography (US) showed a severe left hydroureteronephrosis and an X-ray revealed an impacted stone of about $13 \mathrm{~mm}$ diameter at the level of the iliac tract of the ureter. Laboratory tests were unremarkable except for increased creatinine up to $1.5 \mathrm{mg} / \mathrm{dL}$. There was no leukocytosis. Ureteroscopy (URS)

Departments of ${ }^{1}$ Gynecology-Obstetrics and Urology and ${ }^{2}$ Clinical Medicine, Sapienza University of Rome, Rome, Italy.

(C) Stefano Salciccia et al. 2018; Published by Mary Ann Liebert, Inc. This is an Open Access article distributed under the terms of the Creative Commons Attribution License, which permits unrestricted use, distribution, and reproduction in any medium, provided the original work is properly cited. 
with holmium laser fragmentation was performed and a Double-J stent was inserted for edema and inflammation of the ureteral walls in the side of the stone. Double-J stent was removed after 15 days. After 30 days from stent removal, the patient referred a mild left flank pain and US showed a moderate hydroureteronephrosis. An X-Urography showed a $4 \mathrm{~cm}$ stenosis of the iliac tract of the left ureter. A kidney scintigraphy showed a normal renal function. In accordance with the patient, it was decided to proceed endoscopically to a balloon dilatation of the stenosis followed by the positioning of an AUS (Fig. 1). At 1-month follow-up, US showed a slight dilation of left renal pelvis (Grade-I) and the same situation was maintained at 3- and 6-month follow-ups. Subsequently the patient was lost in the follow-up for severe cardiovascular problems. He came back again to our department after 2 years from the last control, without any sign or symptom. An US showed a severe left hydroureteronephrosis with a marked thinning of the renal parenchyma and a secondary stone of about $15 \mathrm{~mm}$. An abdomen X-ray showed the AUS in the original position. A kidney scintigraphy was planned and it showed the complete loss of left kidney function. Considering the general conditions of the patient (ASA score IV) and the lack of symptoms, we decided to follow the patient at regular intervals and to be operated only in case of complications.

\section{Discussion}

Ureteral strictures are a recurrent chronic condition that leads to severe side effects and poor quality of life. Management of ureteral strictures is a great challenge for urologists and no specific guidelines exist.

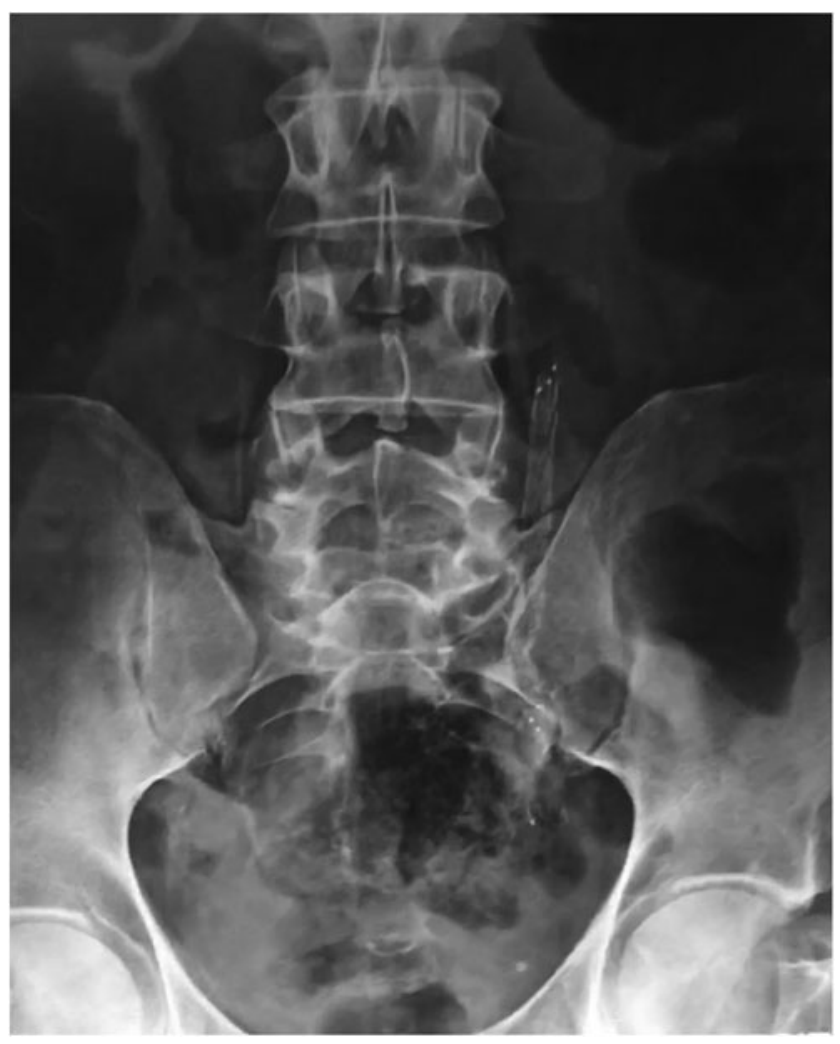

FIG. 1. Abdomen X-ray showing Allium ${ }^{\circledR}$ ureteral stent positioning in the iliac tract of the left ureter.
There are several options, including repeated dilatations, stents, minimally invasive reconstructive surgery, and urinary diversion or nephrectomy. AUS is a new device recently introduced to treat ureteral and urethral stenosis. Considering the recent introduction into the clinical practice, we have only few studies on AUS for ureteral strictures and mainly we do not have specific indications on when to remove the AUS. Moreover, another open question is whether the AUS can be considered a permanent device, for example, in the management of ab-estrinseco compression of the ureter from oncologic diseases. Since 2014, we have treated $>50$ cases of urethral stenosis with AUS, obtaining good functional results. We believe that the optimal time to remove the stent should be between 6 and 12 months. We reported a case in whom after 36 months of Allium positioning, it developed a severe hydronephrosis with a complete loss of left kidney function probably because of the formation of a long acalasic stretch of the ureter in the side of the AUS. We can hypothesize that the presence of the stent in the wall of the ureter for a long period of time can trigger inflammation and a subsequent fibrosis with loss of normal ureteral peristalsis. Another hypothesis is that in our case, the stenosis was very severe and the marked dilation produced a stripping and tearing of the fibromuscular component of the ureter, resulting in fibrosis and loss of physiologic peristaltic function. Unfortunately, we did not have the possibility to perform an URS at a shorter follow-up from the procedure to confirm this second hypothesis. Some studies ${ }^{5-7}$ have reported stent migration or infection as complications of AUS. We believe that the development of ureteral achalasia should also be considered as a possible complication of AUS.

\section{Conclusions}

AUS is a new and promising device for the treatment of ureteral stenosis. Some studies have reported promising results for the treatment of ureteral stenosis. However, there is a lack of standardization of the technique and predictive factors of success or complications have not been determined. For these reasons, we recommend a close instrumental follow-up in the early months after the procedure so to decide the optimal removal time of the stent or to identify a progressive hydronephrosis as a marker of possible failure.

\section{Disclosure Statement}

No competing financial interests exist.

\section{References}

1. Legemate JD, Wijnstok NJ, Matsuda T, et al. Characteristics and outcomes of ureteroscopic treatment in 2650 patients with impacted ureteral stones. World J Urol 2017; 35:1497-1506.

2. Robert WW, Caddedu JA, Fabrizio MD, et al. Ureteral stricture formation following removal of impacted calculi. J Urol 1999;161 Suppl:376.

3. Fam XI, Singam P, Ho CC, et al. Ureteral stricture formation after ureteroscope treatment of impacted calculi: A prospective study. Korean J Urol 2015;56:63-67.

4. Tran H, Arsovska O, Paterson RF, et al. Evaluation of risk factors and treatment options in patients with ureteral stricture disease at a single institution. Can Urol Assoc J 2015;9: E921-924. 
5. Bahouth Z, Meyer G, Halachmi S, et al. Multicenter experience with allium ureteral stent for the treatment of ureteral stricture and fistula. Harefuah 2015;154:753-756, 806.

6. Guandalino M, Droupy S, Ruffion A, et al. The Allium ureteral stent in the management of ureteral stenoses, a retrospective, multicenter study. Prog Urol 2017;27:26-32.

7. Papatsoris AG, Buchholz N. A novel thermo-expandable ureteral metal stent for the minimally invasive management of ureteral strictures. J Endourol 2010;24:487-491.

Address correspondence to: Stefano Salciccia, $M D$ Department of Gynecology-Obstetrics and Urology Sapienza University of Rome Rome 00161 Italy

E-mail: stefano.salciccia@uniroma1.it

$\begin{aligned} & \text { Abbreviations Used } \\ \text { AUS } & =\text { Allium }{ }^{\circledR} \text { ureteral stent } \\ \mathrm{US} & =\text { ultrasonography } \\ \mathrm{URS} & =\text { ureteroscopy }\end{aligned}$

Cite this article as: Salciccia S, Sciarra A, Polese M, Giorgio A, Maggi M, Gentilucci A, Ciccariello M, D’Eramo G, Shahabadi H, Lai S, Ricciuti GP (2018) Loss of renal function after retrograde ureteral placement of an Allium stent for severe ureteral stricture, Journal of Endourology Case Reports 4:1, 9-11, DOI: 10.1089/cren.2017.0117. 